\title{
Surgical interventions in intracranial arteriovenous malformations: Indications and outcome analysis in a changing scenario
}

\author{
Amit Thapa, P. Sarat Chandra, Sumit Sinha, Aditya Gupta, Manmohan Singh, Ashish Suri, B. S. Sharma \\ Department of Neurosurgery, All India Institute of Medical Sciences, Ansari Nagar, New Delhi - 110 029, India
}

\author{
Address for correspondence: \\ Dr. P. Sarat Chandra, \\ Department of Neurosurgery, Room 607, \\ Neurosciences Faculty Chambers, $6^{\text {th }}$ Floor, \\ CN Center, All India Institute of Medical \\ Sciences, New Delhi - 110 029, India. \\ E-mail: saratpchandra@aiims.ac.in/ \\ saratpchandra@gmail.com
}

\begin{abstract}
Background: Intracranial arteriovenous malformations (AVM) are being increasingly managed by multimodality approach. This changing scenario encouraged us to study the present state of surgery in intracranial AVMs and the outcomes. Materials and Methods: Of a total of 868 patients evaluated for suspected or known AVMs between January 2000 and July 2008, 790 had intracranial AVMs. The clinical characteristics and surgical outcomes of the 111 opeated patients were analyzed. Results: Of the 111 patients, 73 were males. Clinical features included: Headache (70\%), loss of consciousness (48\%) and seizures (32\%). The commonest AVM grade was Spetzler-Martin (SM) grade II (41\%), 7\% had AVM $>6 \mathrm{~cm}$ and $78 \%$ had evidence of bleed. In total 143 surgeries were performed and 22\% of patients required multiple interventions. The types of surgical interventions included elective excision of AVM in 23\%, emergency surgery (either AVM excision or evacuation of hematoma) in $55 \%$, surgery following radiosurgery/embolization in $5 \%$ and palliative non-definitive surgeries (e.g. shunt) in 15\%. Post-operative angiography was done in $67 \%$ of patients. Obliteration rates for elective excision of AVM in Spetzler Martin Grade I, II, IIIa, IIIb and IV were 100\%, 7I\%, 33\%, 50\% and 67\% respectively (mean follow-up:31.6 months). Of 39 patients with residual AVMs, 33 received gamma knife and four underwent embolization. Outcome was modified Rankin scale (mRS) grade 1 in 34\% of paitnets and the overall favorable outcome was $83 \%$ and there were six deaths. Conclusion: In our patients' cohort one in every eight patients required surgery. In intracranial AVMs, surgery still plays an important role. In developing countries like India it may be beneficial to electively excise Grade I and II AVMs if cost is a consideration.
\end{abstract}

Key words: Intracranial arteriovenous malformations, surgery, surgical intervention, surgical outcome

\section{Introduction}

The reported incidence of intracranial arteriovenous malformation (AVM) is about 1 per 100000 per year and the point prevalence in adults is 18 per 100000 . AVMs account for $1-2 \%$ of all strokes, $4 \%$ of intracerebral hemorrhages and $9 \%$ of subarachnoid hemorrhages.
In young adults AVMs account for one-third of the etiology of intracerebral hemorrhages. ${ }^{[1]}$ With widespread availability of adjuvant treatment modalities like gamma knife radiosurgery, the role of surgery in intracranial AVM is becoming limited. This changing trend encouraged us to study the treatment patterns in AVMs and also to study the place of surgery in this changing scenario at our center 
where all three modalities to manage intracranial AVMs are available.

\section{Materials and Methods}

Records of patients with AVM attending Neurosciences Centre, All India Institute of Medical Sciences, New Delhi between January 2000 and July 2008 were retrieved. Patients with diagnosis of intracranial AVM (based on Magnetic Resonance Imaging (MRI)/Magnetic Resonance Angiography (MRA)/CT Angiography/Intraarterial Digital Subtraction Angiography (IADSA), who underwent surgery for intracranial AVM were included in this study. Intracranial AVMs were classified using Spetzler and Martin grading. Surgical excision of AVM was carried out using standard microneurosurgical techniques. The outcomes were analyzed according to modified Rankin Scale (mRS). ${ }^{[2,3]}$ Chi-square test was used to compare treatment offered and the outcomes in each Spetzier Martin grade. Data was analyzed using SPSS, Version 14.0 (SPSS).

\section{Results}

During the study period of the 888 patients investigated,
790 were found to have intracranial AVMs. One hundred eleven of them, one in every eight patients, underwent surgical interventions [Table 1]. There were $74(66.7 \%)$ males, age range was 7-67 years (mean 28.7$)$. There was one pregnant female in early third trimester. Associated morbidities included hypertension (one) and pulmonary tuberculosis (one). Of the 111 patients, $103(92.8 \%)$ patients were symptomatic at the time of presentation, acute onset symptoms due to intracerebral hemorrhage in $50(45 \%)$ patients. Commonest presenting complaint was headache $(70 \%)$ followed by history of loss of consciousness (48\%) and convulsive seizures $(32 \%)$. The headache was sudden in onset in $65 \%$ of patients and the location of headache did not correspond to the location of AVM. Two-thirds of the patients with seizures had multiple seizures. Seizure type was generalized tonic-clonic in 27 patients, focal with secondary generalization in four, focal in three and status in one. Focal deficits included dysphasia (2), visual field defects (3), facial nerve palsy (7), VI nerve palsy (1) and cerebellar signs (3).

Eighty-four patients (75.7\%) presented with intracranial bleed: Intracerebral in 69 (82\%), intraventricular in 27 $(32 \%)$, subarchnoid in six $(7 \%)$ and subdural in two

\section{Table 1: Characteristics of the study group $(n=111)$}

Epidemiology

Symptoms

Physical examination

Radiology

\section{Surgical intervention}

Number of surgery

$[n=143]$

Indication for re-operation

[n $=24]$

Operation $[n=143]$

Postoperative multimodality treatment $[n=37]$

Intra/postoperative Complications $[n=61]$ Outcome

Duration of follow-up Angiographic obliteration* Modified rankin score at last follow-up
Age (mean, range in years): 28.7, 7-67; Sex (Male: Female): 74:37

Headache: 77 (70\%); loss of consciousness: 53 (48\%); convulsions: 35 (32\%); vomiting: 35 (32\%); limb weakness: 28 (25\%); loss of vision: 7 (6\%); dysarthria-diplopia: 8 (7\%); asymptomatic: 8 (7\%)

Glasgow coma scale <8: 13 (12\%); 9-13:8 (7\%); 14-15: 90 (81)

Altered sensorium: 47 (42\%); dysphasia: 2 (2\%); visual field defects: 3 (3\%); CN VI-VII palsy: 8 (7\%)

Location- Frontal:34 (31\%);Temporal:16 (14\%);Parietal:10 (9\%); Parieto-Occipital:7 (6\%); Occipital: 5 (5\%);

Fronto-parietal: 5 (5\%);Temporo-parietal: 5 (5\%); Basal Ganglia: 4(4\%); Choroidal: 2 (2\%); Striatal: 2 (2\%);TemporoOccipital: 1 (1\%); Parieto-occipital-Temporal: 1 (1\%); Callosal: 1 (1\%); Trigonal: 1(1\%); Cerebellar:14 (13\%);

Brainstem: 1 (1\%)

Spetzler Martin grade I: 15 (14\%); II: 45 (40\%); Illa: 10 (9\%); Illb: 21 (19\%);IV: 18 (16\%);V: 2 (2\%)

Size of AVM $<3 \mathrm{~cm}: 51$ (46\%); 3-6 cm: 52 (47\%); $>6 \mathrm{~cm}: 8$ (7\%)

Location of bleed- Lobar: 46 (55\%); Lobar with Intraventricular bleed (IV) extension: 17 (20\%);IV bleed: 8 (10\%); Subarachnoid hemorrhage (SAH): 6 (7\%); Lobar with SAH: 3 (4\%); all three: 2 (2\%); Subdural bleed: 1 (1\%); lobar with subdural bleed: 1 (1\%)

Associated anomaly- Aneurysm: 13 (12\%); Cavernoma: 1 (1\%)

Elective AVM excision: 25 (22.5\%); Emergency AVM Excision/hematoma evacuation: 61 (55\%); AVM excision following failed embolization/staged Rx: 5 (4.5\%); other surgery: 20 (18\%)

1: $87(78 \%) ; 2: 17(15 \%) ; 3: 6(5 \%) ; 4: 1(1 \%)$

CSF diversion: 10 (42\%); staged Rx: 5 (21\%); Postoperative hematoma: 3 (13\%); Brain edema: 1 (4\%); Cranioplasty: 1(4\%); Clipping of aneurysm: 1(4\%); Infected bone flap: 1(4\%)

AVM excision: 57 (40\%); Hematoma evacuation: 30 (21\%); lobectomy only: 8 (6\%); AVM excision + Aneurysm

Clipping: 6 (4\%); Decompressive craniectomy: 4 (3\%); exploration: 3 (2\%); aneurysm Clipping: 3 (2\%);

Lobectomy + Decompressive craniectomy: 1(1\%);EVD:5(3\%); Shunt:13(9\%); EVD + ommaya:3(2\%); EVD + Shunt: $1(1 \%)$

GKRS following hematoma evacuation: 14 (38\%); GKRS following AVM excision: 8 (22\%); GKRS following CSF diversion: 8 (22\%); Embolization following AVM excision or hematoma: 2 (5\%); Embolization following CSF diversion: 2 (5\%); GKRS following surgical excision and embolization: $3(8 \%)$

Intraoperative rupture: 1(2\%); Non-localization: 2 (3\%); Residual AVM: 30 (49\%); Sepsis: 5 (8\%); Shunt malfunction:

4 (7\%); Seizure: 3 (5\%); Hematoma: 3 (5\%); Meningitis:3(5\%); Dysphasia: $2(3 \%)$

Improved: 100(90\%); Deteriorated: 5 (5\%); Expired: 6(5\%)

$<1$ month: 23 (21\%); 1 month -1 year: $43(39 \%)$; >1 year: $45(41 \%)$

Following AVM excision: 19/34; Lobectomy: 1/1; Hematoma excision: $9 / 21$

0:38(34\%); 1:23 (21\%); 2: 17 (15\%); 3: 14 (13\%); 4:4 (4\%); 5:9 (8\%); 6: 6 (5\%)

*Denominator represents total number of patients undergoing check angiography 
(2\%). Associated hydrocephalus was seen in $21(25 \%)$ patients and 22 of them required cerebrospinal fluid (CSF) drainage procedures (ventriculoperitoneal shunt, Ommaya reservoir placement, and external ventricular drainage).

Location of AVM was supratentorial in $85.6 \%$ (frontal in $32.4 \%$ and temporal in $14.4 \%$ ) cerebellar in $12.6 \%$ and brainstem 1\%. Spetzler Martin (SM) grading of AVM was: SM Grade II: 40\%; SM Grade III b:19\%, SM Grade IV: $16 \%$ and SM Grade V: $2 \%$ The AVM was $<3 \mathrm{~cm}$ diameter in $51(46 \%)$ patients, 3-6 cm in $52(47 \%)$ patients and $>6 \mathrm{~cm}$ in eight $(7 \%)$ patients. Majority of patients with intracranial hemorrhage had SM Grade II AVMs. Associated anomalies on angiography included aneurysms $(11.7 \%)$ and cavernomas $(0.9 \%)$.

\section{Surgical intervention}

One hundred and eleven patients underwent a total of 143 operations. Twenty-four patients $(22 \%)$ required multiple surgeries: Two operations in $17(15 \%)$, three operations in six $(5 \%)$, and four operations in one (1\%). Based on the types of surgeries patients had, they were divided into four groups:

\section{Group I - Elective surgical excision of arteriovenous malformations}

Elective microsurgical excision of the AVM was done in 25 patients $(22.5 \%)$. The indications for surgery were surgical accessibility (64\%), superficial location Spetzler Martin Grade I/II AVM (44\%), and location in noneloquent cortex $(48 \%)$. Five $(4.5 \%)$ patients required aneurysm clipping at the same sitting. Check angiogram was done in 16 patients, nine (56.3\%) had complete obliteration of the AVM. The major complications included expressive aphasia (8\%), seizures (4\%), and chest infection (8\%). Most of the patients in this group could not afford other modes of interventions because of limited resources.

\section{Group II - Emergency surgical procedure}

61 patients (55\%) underwent emergency operative procedures like hematoma evacuation or excision of AVM. Of the 61 patients in this group 57 (93.4\%) had intracerebral hematoma. AVM grading was: SM Grade II-25 (41\%), SM Grade IIIb-12 (19.7\%) and SM Grade IV$12(19.7 \%)$. Check angiogram was done in 37 patients, $18(48.7 \%)$ had complete obliteration of the AVM. The complications included operative site hematoma (4.9\%) and chest infection (3\%).

Group III: Elective excision of arteriovenous malformations as a staged therapy

Five $(4.5 \%)$ patients had elective surgery for the excision of AVM as a staged therapy after embolization and/ or Gamma Knife Radiosurgery (GKRS). The grade of AMVs was SM Grade II and 60\% of AVMs were located in eloquent cortex. Four patients who had preoperative embolization, developed intracranial bleed, three during the procedure and one in follow-up period. All these patients were initially managed conservatively and later subjected to planned AVM excision. Of the three patients who had check angiogram, one had complete obliteration of the AVM. One patient each had postoperative seizure and operative site hematoma.

\section{Group IV: Palliative surgical procedure}

Twenty patients (18\%) had surgical procedures unrelated to AVM excision or hematoma evacuation, like cerebrospinal fluid (CSF) diversion procedure (ventriculoperitoneal shunt, Ommaya reservoir placement, external ventricular drainage) and decompressive craniectomy. AVM grade was: SM Grade II-8 (40\%), SM Grade III b-6 (30\%), SM Grade IV-2 (10\%), and SM Grade V-1 (5\%). Associated hydrocephalus was seen $17(85 \%)$ of patients. Of the 14 patients who had check angiogram, three had complete obliteration of the AVM. Three patients (15\%) had shunt malfunction.

Various surgical procedures done in the 111 patients included: Microsurgical excision of AVM (40\%), evacuation of hematoma (21\%), clipping of associated aneurysm (5\%), lobectomy for AVM and hematoma removal $(6 \%)$, decompressive hemicraniectomy $(3 \%)$, and only surgical exploration without excision of AVMs as they were not excisable (3\%). Different treatment modalities offered to patients based on the Spetzler Martin grading of AVMs is given in Figure 1.

Intraoperative complications included: AVM rupture at surgery (1), surgeon's inability to define the limits of AVM at surgery (1). Post operative complications included: Chest infection (6.1\%), seizures (4.5\%), shunt malfunctions $(4.5 \%)$, and wound infection $(2 \%)$. Reoperation was done in 24 patients: [Figure 2] CSF diversions (10), initial evacuation of hematoma and later excision of AVM (4), operative site hematoma evacuation (3), residual AVM exicison (2), decompressive hemicraniectomy (1), aneurysm clipping (1), and infected bone flap removal (1).

Of the 39 patients who had residual AVM, 37 patients turned up for further treatment. Thirty-three received postoperative GKRS and four received postoperative embolization. One patient who required hematoma evacuation, was given two sittings of GKRS with five year interval.

\section{Outcome}

The mean period of follow-up was 31.36 months and maximum 360 months. About $41 \%$ of patients had follow-up one year of surgical treatment. Of the 111 patients, $100(90 \%)$ patients had clinical improvement 


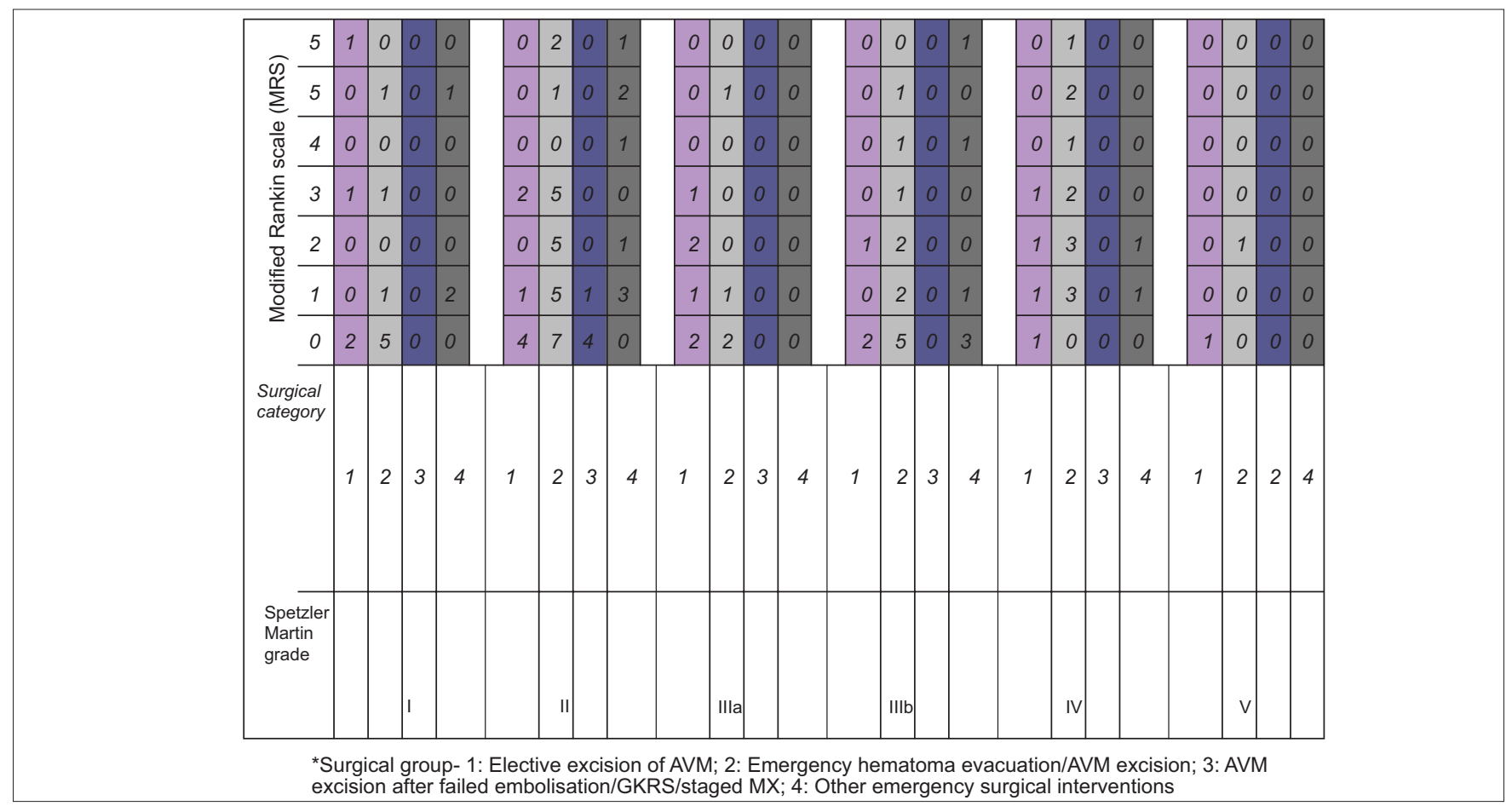

Figure 1: Outcome (Modified Rankin scale) in each Spetzler Martin grade of AVM (roman numbers), which has been sub-divided into 4 groups of surgical interventions. Italicized number in box represents number of patients. In Spetzler Martin grade I, II and Illa, elective excision of AVM had better outcome (MRS < 3) than emergency excision of hematoma/ AVM excision. In higher grades of AVM, emergency surgery were more warranted; however, elective excision of AVM still had good outcome (MRS $<3$ ) as compared to emergency surgery. Overall $83 \%$ patients had good outcome (MRS $<3$ ).

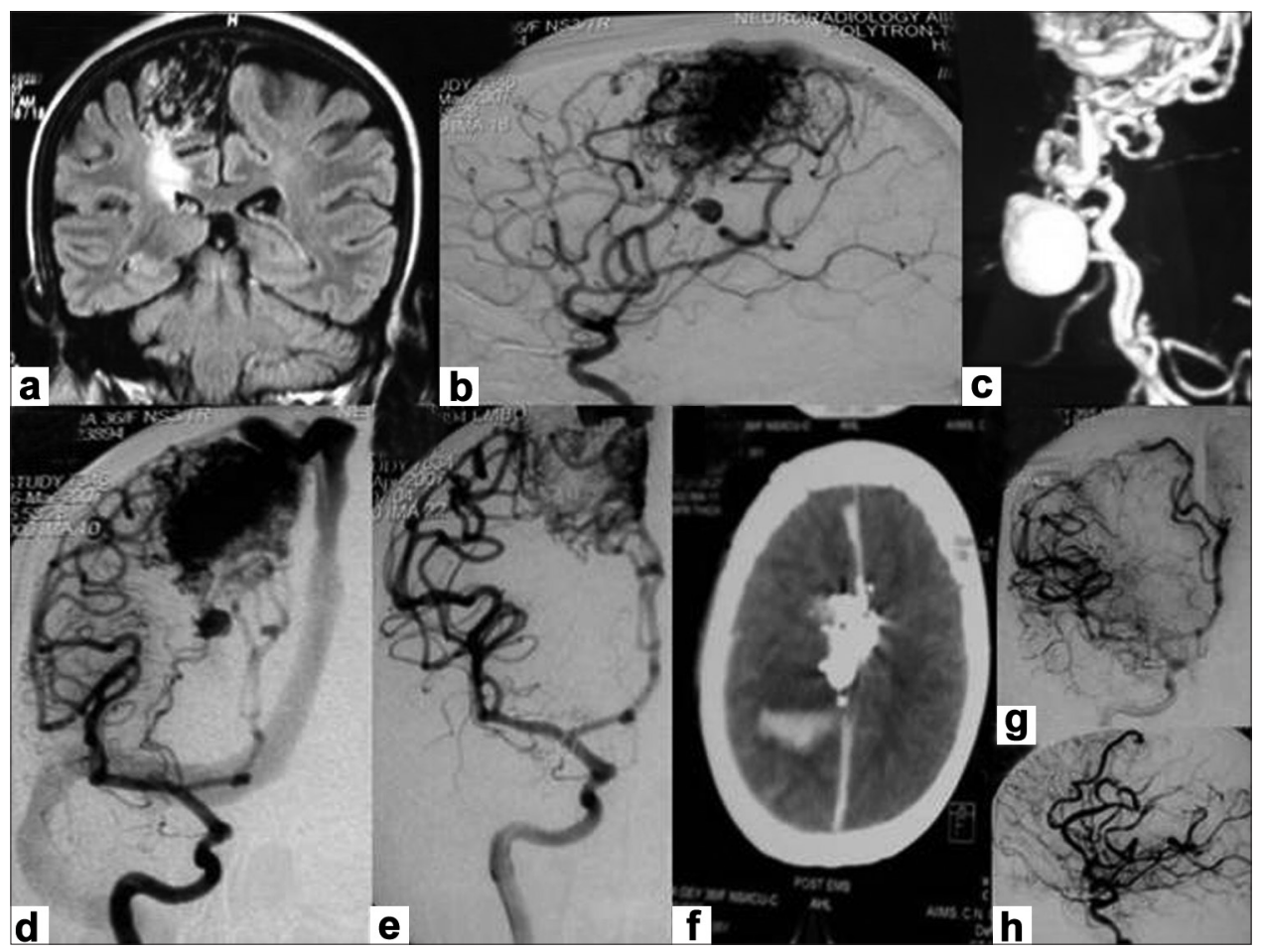

Figure 2: 55 year female with large medial posterior frontal and parietal AVM (a) feeders from MCA and lenticulostriate branches (b, d) draining into the superior saggital sinus. Angiogram (b, d) and 3-D DSA showed a flow related aneurysm on the lenticulostriate branch. (c) Following the clipping of lenticulostriate branch, patient had infarct (e). 9 days later, she underwent an elective embolization, during which she developed a perfusion pressure break through bleed (f). Emergency evacuation of hematoma and excision of AVM was done. Repeat DSA showed total excision of the AVM (g, h).

and $5(4.5 \%)$ deteriorated and discharged home with morbidity, and in hospital deaths were six (5.5\%).
At the last follow-up, 70 (67\%) had check angiogram. The remaining patients were lost for follow-up. In 50\% 
of the patients the check angigram showed complete obliteration of the AVM. Of the 63 patients who had excision of AVM, 34 (55.6\%) had complete obliteration of AVM: $71 \%$ in SM Grade II, 33\% in SM Grade IIIa, 50\% in SM Grade IIIb, and 67\% in SM Grade IV. None of the patient with SM Grade I AVM had check angiogram.

Of the 15 patients with residual AVM who received GKRS, embolization, or both only one showed complete obliteration of the AVM at the last follow-up. Nine $(43 \%)$ of the 30 patients who had hematoma evacuation had complete obliteration of AVM. Of this subtype of 30 patients, 14 patients received postoperative GKRS, four of them demonstrated complete obliteration of the $\mathrm{AVM}$ at the last follow-up.

Functional outcome at the last follow-up was mRS $<3$ in $92(82.9 \%)$ patients. Significant morbidity was present in $11.7 \%$ of patients and $5 \%$ of patients who presented in poor grades died. Significant improvement in various AVM SM gradings was: SM Grade 1-12 (80\%); SM Grade II-38 (84.4\%); SM Grade IIIa-17 (81\%). SM Grade IIIb-14 (77.8\%), SM Grade IV-2. Functional outcome (MRS) in each treatment group based on Spetzler Martin grading is given Figure 1. The outcomes were better in patients with SM Grades I, II, and IIIa who had excision of the AVM. All the six patients with in hospital mortality were in poor GCS score, $<8$. The cause of death was severe sepsis with multiorgan failure in three, meningitis in one, and cerebral edema and herniation in two.

\section{Giant arteriovenous malformations}

In eight $(7 \%)$ patients the size of the AVM was more than $6 \mathrm{~cm}$ in diameter and seven $(87.5 \%)$ of them had good outcome. One of the patient who had CSF drainage procedure for hydrocephalus later received GKRS. Two patients were operated for hematoma evacuation and the remaining five patients had excision of the AVM. Of these patients, one patient was taken for preoperative embolization but had breakthrough bleeding and had to be re-operated for hematoma evacuation. One patient had postoperative hemiparesis and two patients had persistence of seizures.

\section{Discussion}

The epidemiology and natural history of intracranial AVM is still incompletely elucidated. AVM usually presents between 20 and 40 years of age. ${ }^{[4]}$ The mean age (28.7 yrs) in our cohort was lower than the mean age (31.238.3 yrs) in other reported series. ${ }^{[4,5]}$ There is male gender preponderance in intracranial AVMs. ${ }^{[5]}$ AVMs are often asymptomatic and the diagnosis is incidental in up to $15 \%$ cases $^{[5]}$ and it was $8 \%$ in our cohort and were detected on contrast CT while patients were being investigated for nonspeciric symptoms. The presenting feautes may be intracranial hemorrhage, headache, seizures, and focal deficits. ${ }^{[1,6]}$ Similar were the presenting features in our cohort. However, our cohort was more symptomatic and is on the expected lines for the socioecomic status of the Indian population. The risk of disability in AVMs is $1 \%$ per year.

The aim of treatment in intracranial AVMs is two folds: (1) to prevent the catastrophic intracranial hemorrhage and (2) to alleviate neurological symptoms with an acceptable risk. Most of the studies suggest that the total reduction in the risk of hemorrhave can only be achieved by the complete obliteration of the AVM. ${ }^{[7]}$ Since the risk of unruptured AVM is much less than the ruptured AVM, treatment of unruptured AVM caries higher risk-to-benefit ratio. This makes the selection of management difficult. ${ }^{[8,9]}$ In the absence of any randomized clinical trials, a standardized treatment algorithm is still elusive and depends on treating physician's specialization and approach. In this regard a trail (ARUBA) is ongoing in patients with unruptured AVMs. Managent approach depends on whether AVM has ruptured or was incidental, neurological status, age of patient, co-morbidities and angio-architecture of the AVM. During the last four decades, all modalities of treatment (surgical excision, embolization and radiosurgery) have undergone considerable refinement in technique and safety.

At our center we have all the facilities to treat intracranial AVMs: GKRS, interventional neuroradiology, and microneurosurgery facilities. Patient management issues are discussed in the neurosurgery-radiology meetings. Patient's socioeconomic status is also taken into account while deciding the treatment approach. In our center the chance of patient having surgery was one in eight. As identified in this study the surgical procedures required in patients with intracranial AVMs can be categorized into four groups. The first group included Spetzler Martin Grade I and II AVMs located in non-eloquent area, where surgical excision was offered as per the choice of the patients. At our centre, the minimal costs of various procedures are: Surgery - INR 20000 (US\$ 400), GKRS - INR 75000 (US\$ 1500) and embolization - NR 100000 (US\$2000). The second group included patients who required emergency evacuation of hematoma with or without excision of AVM. The third category usually included Grade IIIb, IV and V AVM who were advised AVM excision after failure of multiple sittings of embolization or GKRS or as a part of staged therapy. The fourth category included palliative surgeries like decompressive craniotomies and CSF drainage procedures. No definitive surgical excision of AVM was done in some patients in this group. Surgery for intracranial AVM has the advantage of immediate and long-term reliable angiographic obliteration of almost $100 \%$ for SM Grade I and II AVMs and is still widely used. The most common indications for surgical 


\begin{tabular}{|c|c|c|c|c|c|c|}
\hline & $\begin{array}{c}\text { Our series } \\
2009 \\
\end{array}$ & $\begin{array}{c}\text { Munoz } \\
\text { et al. }{ }^{[10]} 2007\end{array}$ & $\begin{array}{c}\text { Zhao } \\
\text { et al. }{ }^{[5]} 2004\end{array}$ & $\begin{array}{c}\text { Chang } \\
\text { et al. }{ }^{[11]} 2003\end{array}$ & $\begin{array}{c}\text { Derut } \\
\text { et al. }{ }^{[12]} 1998\end{array}$ & $\begin{array}{c}\text { Schaller } \\
\text { et al. }{ }^{[13]} 1996\end{array}$ \\
\hline Number of patients & 790 & 31 & 2086 & 53 & 90 & 126 \\
\hline Mean age (years) & 28.7 & 31.6 & 38.3 & & & 34.7 \\
\hline \multicolumn{7}{|l|}{ Presentation (\%) } \\
\hline Intracranial hemorrhage & 78 & & 43.4 & $20(38)$ & & 37.3 \\
\hline Seizure & 32 & 12.9 & $17.3 \%$ & $18(34)$ & & 34.9 \\
\hline Ischemic event & Nil & 3.2 & & $7(13)$ & & \\
\hline Incidental finding & 7 & 3.2 & & & & \\
\hline \multicolumn{7}{|l|}{ Spetzler- martin grading (\%) } \\
\hline Grade I & 14 & 10.3 & 20.4 & & 68 & 20.6 \\
\hline Grade II & 40 & 24.1 & 43.7 & & & 28.6 \\
\hline Grade III & 28 & 37.9 & 27.3 & 1 & & 32.5 \\
\hline Grade IV & 16 & 24.1 & 7.6 & 9 & 32 & 14.3 \\
\hline Grade V & 2 & 3.4 & 1 & 43 & & 4 \\
\hline \multicolumn{7}{|l|}{ Treatment } \\
\hline Surgery & 111 & 6 & 1255 & 27 & & 126 \\
\hline Preoperative embolization & 5 & 5 & 77 (intraoperative embolization) & 5 & & 17 \\
\hline Mortality (\%) & $6(5)$ & 1 & $116(9.2)$ & $8(15)$ & & 1 \\
\hline Morbidity (\%) & 19.7 & & $267(21.2)$ & & & 27 to 83 \\
\hline \multicolumn{7}{|l|}{ Modified rankin scale (\%) } \\
\hline 0 & $38(34)$ & $7(36.8)$ & & $27(51)$ & & \\
\hline 1 & $23(21)$ & & & & & \\
\hline 2 & $17(15)$ & $10(52.6)$ & & $15(28)$ & & \\
\hline 3 & $14(13)$ & 0 & & & & \\
\hline 4 & $4(4)$ & $1(5.3)$ & & $3(6)$ & & \\
\hline 5 & $9(8)$ & & & & & \\
\hline 6 & $6(5)$ & 1 & $116(9.2)$ & $8(15)$ & & 1 \\
\hline
\end{tabular}

intervention in our study were intraparenchymal bleed and also patient's choice. Planned definitive surgery following initial hematoma evacuation did not have any worse outcome. But definitive surgery in patients with poor general condition was associated with poor outcomes. In our cohort an average obliteration rates seen in all grades of AVMs was $56 \%$.

The mean moratlity in different surgical series was 9.2\% [Table 2] $\cdot^{[5,10-13]}$ The immediate mortality in our chohort was much less (5\%) and this low mortality could be related to the use of refined microsurgical technique, neuronavigation and good intensive care both peri-operative and postoperatve. The reported frequency morbidity in various series varied from 1.5 to $18.7 \% .{ }^{[5,14]}$ The frequency of various morbidities seen in our cohort were similar to the observed frequencies in other surgical series. ${ }^{[5,14]}$

The probability of favorable outcome in various AVM grades, Spetzler Martin grading is $100 \%, 93 \%$, $83 \%$, $70 \%$, and $53 \%$ in Grade I, II, III, IV and V respectively. ${ }^{[5]}$ Hamilton and Spetzler ${ }^{[15]}$ reported $<1 \%$ mortality and morbidity with resection in Grade I and II AVMs and $<3 \%$ for Grade III AVMs. The morbidity for Grade IV and V AVMs was 31\% and 50\% respectively. We have not analyzed our data on these lines, however, MRS was $<3$ in $80 \%$ in patients of all SM grades. However, this finding has limitation as the number of Patients in higher AVM grades were fewer. The other limitation was slection bias for higher grades. Overall, we found that $82.9 \%$ of our patients achieved significant improvement in functional ability.

Of the 65 patients with AVMs treated by GKRS, 51\% of patients became seizure free and $61 \%$ of patients with intractable seizures had excellent outcome (nondisabling simple partial seizures only) ${ }^{[16]}$ Similar were the observations in our cohort. In our cohort $75 \%$ of patients with seizures had seizure freedom following surgical excision and $16 \%$ had decrease in seizure frequency. We suggest that patients with SM Grade I and II AVMs should preferably have surgery, as the surgery is associated with lower mortality and morbidity. Patients with SM Grade III AVMs would require pre/ intraoperative embolization. Operative risk in patients with SM Grade IV and V AVMs exceeds the risk of bleed ( $<1 \%$ per year) ${ }^{[15,17]}$ It is recommended that these patients should carefully be watched under close follow-up and surgical intervention is advised if the patient develops fresh neurologic deficits or progressive deficits due to repeated bleeds or develops intractable epilepsy. Partial obliteration of the AVM only adds to the risk of re-bleed rather than preventing the complications.

\section{References}

1. Al-Shahi R, Warlow C. A systematic review of the frequency and prognosis of arteriovenous malformations of the brain in adults. Brain 2001;124:1900-26.

2. Rankin J. Cerebral vascular accidents in patients over the age of 60 . Scott Med J 1957;2:200-15. 
3. Bonita R, Beaglehole R. Modification of rankin scale: Recovery of motor function after stroke. Stroke 1988;19:1497-500.

4. Hofmeister C, Stapf C, Hartmann A, Sciacca RR, Mansmann U, TerBrugge K, et al. Demographic, morphological, and clinical characteristics of 1289 patients with brain arteriovenous malformation. Stroke 2000;31:1307-10.

5. Zhao J, Wang S, Li W. Clinical characteristics and surgical results of 2086 patients with cerebral arteriovenous malformation. Int Congr Series 2004;1259:235-42.

6. Mattle HP, Schroth G, Seiler RW. Dilemmas in the management of patients with arteriovenous malformations. J Neurol 2000;247:917-28.

7. Florella D, Albuquerque FC, Woo HH, McDougall CG, Rasmussen PA. The role of neuroendovascular therapy for the treatment of brain arteriovenous malformations. Neurosurgery 2006;59:163-77.

8. Hartmann A, Mast H, Choi JH. Treatment of arteriovenous malformations of the brain. Curr Neurol Neurosei Rep 2007;7:28-34.

9. Stapf C, Mohr JP, Choi JH, Hartmann A, Mast H. Invasive treatment of unruptured brain arteriovenous malformations is experimental therapy. Curr Opin Neurol 2006;19:63-8.

10. Munoz F, Clavel P, Molet J. Current management of arteriovenous malformations: Retrospective study of 31 cases and literature review. Neurocirugia (Astur) 2007;18:394-404.

11. Chang SD, Marcellus ML, Marks MP, Levy RP, Do HM, Steinberg GK.
Multimodality treatment of giant intracranial arteriovenous malformations. Neurosurgery 2003;53:1-11.

12. Deruty R, Pelissou-Guyotat I, Morel C, Bascoulergue Y, Turjman F. Reflections on the management of cerebral arteriovenous malformations. Surg Neurol 1998;50:245-55.

13. Schaller C, Pavlidis C, Schramm J. Differential therapy of cerebral arteriovenous malformations: An analysis with reference to personal microsurgery experiences. Nervenarzt 1996;67:860-9.

14. Castel JP, Kantor G. Postoperative morbidity and mortality after microsurgical exclusion of cerebral arteriovenous malformations Current data and analysis of recent literature. Neurochirurgie 2001:47:369-8

15. Hamilton MG, Spetzler RF. The prospective application of a grading system for arteriovenous malformations. Neurosurgery 1994;34:2-7.

16. Schäuble B, Cascino GD, Pollock BE, Gorman DA, Weigand S, CohenGadol AA, et al. Seizure outcomes after stereotactic radiosurgery for cerebral arteriovenous malformations. Neurology 2004;63:683-7.

17. Han PP, Ponce FA, Spetzler RF. Intention-to-treat analysis of SpetzlerMartin grade IV and V arteriovenous malformations: Natural history and treatment paradigm. J Neurosurg 2003;98:3-7.

Accepted on 16-08-2009

Source of Support: Nil, Conflict of Interest: None declared.

\section{Dispatch and return notification by E-mail}

The journal now sends email notification to its members on dispatch of a print issue. The notification is sent to those members who have provided their email address to the association/journal office. The email alerts you about an outdated address and return of issue due to incomplete/incorrect address.

If you wish to receive such email notification, please send your email along with the membership number and full mailing address to the editorial office by email. 